\title{
Miliary lung metastases from non-small cell lung cancer with Exon 20 insertion: A dismal prognostic entity: A case report
}

\author{
AKIMASA SEKINE $^{1}$, TAKUMA KATANO ${ }^{1}$, TSUNEYUKI ODA ${ }^{1}$, SATOSHI IKEDA ${ }^{1}$, TAE IWASAWA ${ }^{2}$, \\ HIROAKI SATOH $^{3}$, TOSHIRO KATAOKA ${ }^{4}$, KOJI OKUDELA ${ }^{4}$ and TAKASHI OGURA ${ }^{1}$ \\ Departments of ${ }^{1}$ Respiratory Medicine and ${ }^{2}$ Radiology, Kanagawa Cardiovascular and Respiratory Center, \\ Yokohama, Kanagawa 236-0051; ${ }^{3}$ Department of Internal Medicine, Mito Medical Center, University of Tsukuba, \\ Tsukuba, Ibaraki 305-8577; ${ }^{4}$ Department of Pathology, Graduate School of Medicine, \\ Yokohama City University, Yokohama, Kanagawa 236-0027, Japan
}

Received March 22, 2018; Accepted July 2, 2018

DOI: $10.3892 / \mathrm{mco} .2018 .1730$

\begin{abstract}
Miliary lung metastases have been reported to be frequently observed in non-small cell lung cancer (NSCLC) with major EGFR mutations, which consist of exon 19 deletion and exon $21 \mathrm{~L} 858 \mathrm{R}$. However, it remains undetermined whether NSCLC with minor EGFR mutations possesses characteristics similar to those with major EGFR mutations. In the present study, two cases of miliary lung metastases from NSCLC with exon 20 insertion were reported. Both the patients visited our hospital because of cough and/or dyspnea and were treated with chemotherapeutic agents, including platinum-doublet regimen. In addition, 1 patient received afatinib during the clinical course. However, all therapeutic regimens did not result in the desired outcome, and the respiratory condition rapidly deteriorated. Both the patients succumbed to disease within 3 months from the beginning of the 1st-line treatment due to disease progression. To conclude, chest physicians should be aware that miliary lung metastases could develop in NSCLC patients with exon 20 insertion and present a dismal prognosis.
\end{abstract}

\section{Introduction}

Epidermal growth factor receptor-tyrosine kinase inhibitors (EGFR-TKIs), including gefitinib, erlotinib, and afatinib, have a dramatic effect on non-small cell lung cancer (NSCLC) with major EGFR mutations consisting of exon 19 deletion and exon 21 L858R point mutation $(1,2)$. More recently, some clinical and radiological characteristics of NSCLC with major EGFR mutations were revealed: Miliary lung metastases are

Correspondence to: Dr Akimasa Sekine, Department of Respiratory Medicine, Kanagawa Cardiovascular and Respiratory Center, Tomiokahigashi 6-16-1, Kanazawa-ku, Yokohama, Kanagawa 236-0051, Japan

E-mail: akimasa.sekine@gmail.com

Key words: exon 20 insertion, lung cancer, miliary metastases frequently observed in NSCLC with EGFR mutation $(3,4)$. In addition, miliary brain metastases have been reported to be correlated with exon 19 deletion $(5,6)$.

However, it remains undetermined whether NSCLC with minor EGFR mutations possesses characteristics similar to those with major EGFR mutations. Exon 20 insertion is reportedly rare and accounts for only 4\% of EGFR mutations (7-9). Between January 2014 and September 2017, we observed four cases of NSCLC with exon 20 insertion. Among them, two patients presented with miliary lung metastases at the time of NSCLC diagnosis. In the present study, we describe notable clinical courses in these two patients.

\section{Case report}

Case 1. A 53-year old non-smoking woman developed cough and visited a hospital on July 12, 2016. A chest radiograph demonstrated multiple pulmonary nodules (Fig. 1A), which suggested miliary tuberculosis. The patient was referred to our hospital on July 13. Chest CT showed numerous miliary nodules throughout bilateral lungs (Fig. 1B and C); however, a sputum smear stain for mycobacteria was negative. Transbronchial lung biopsy on July 19 revealed papillary adenocarcinoma staged cT4N3M1b (PUL, BRA) and cobas version 2 method detected exon 20 insertion. Because the performance status (PS) score was zero, cisplatin plus pemetrexed were administered as first-line therapy on August 16. However, pleural effusion and multiple lung nodules deteriorated after two-courses of this regimen. On September 9, docetaxel plus ramucirumab were administered as second-line treatment. However, the patient developed lymphangitis with deterioration of her respiratory condition. After methylprednisolone was administered to treat the lymphangitis on September 17 (Fig. 1D), afatinib was administered as third-line chemotherapy on September 19th. Although the patient exhibited temporary improvement in her respiratory condition on 30th September from required oxygen of 15 to $1 \mathrm{l} / \mathrm{min}$ (Fig. 1E), the lesions in the right lower lung deteriorated and progression of disease was confirmed on October 24 (Fig. 1F). Subsequently, nivolumab was administered on October 25, but it failed to produce the desired outcome. Finally, she died of lung cancer on November 2 . 


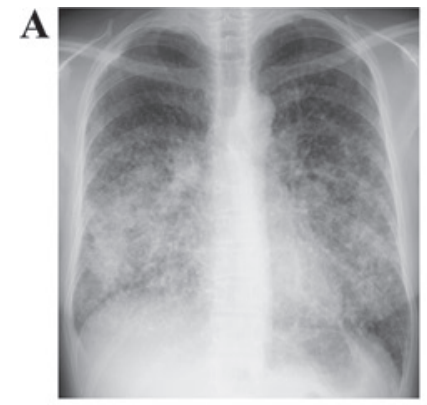

$B^{-}$

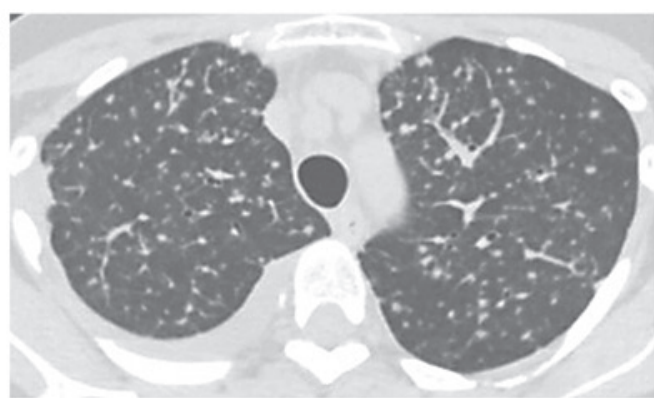

C

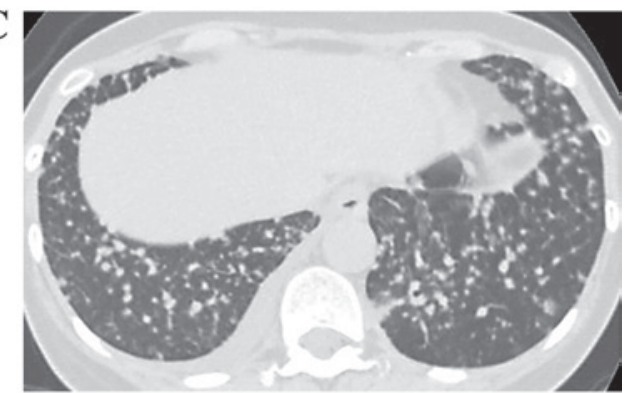

D

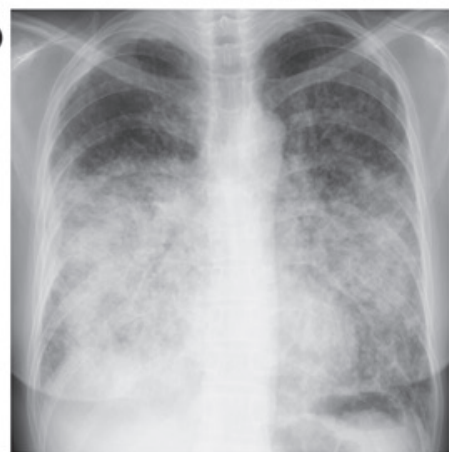

$\mathbf{E}$

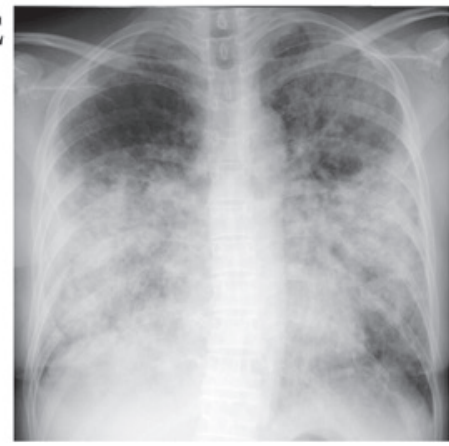

$\mathbf{F}$

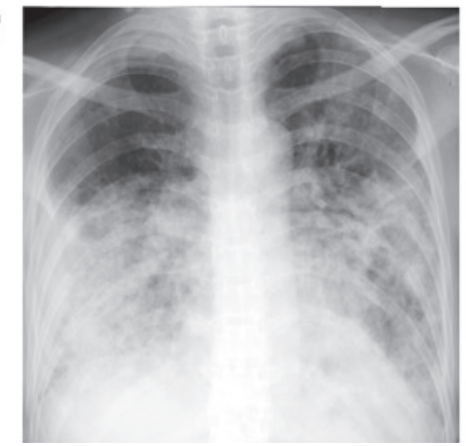

Figure 1. (A) Chest radiograph showed multiple nodules bilaterally in the lungs. (B and C) Chest CT on admission demonstrated numerous tiny nodules bilaterally in the lungs. (D) Chest radiograph before steroid and afatinib treatment showed extensive consolidations bilaterally in the lungs. (E) On September 27, right lung consolidations slightly improved, although left lung lesions deteriorated. (F) On October 23, extensive consolidations progressed especially bilaterally in the lower lung fields.

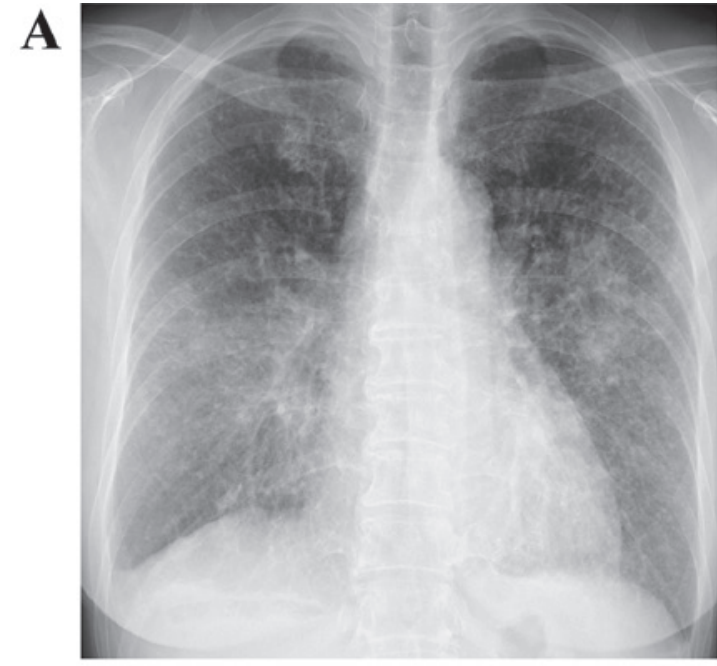

C

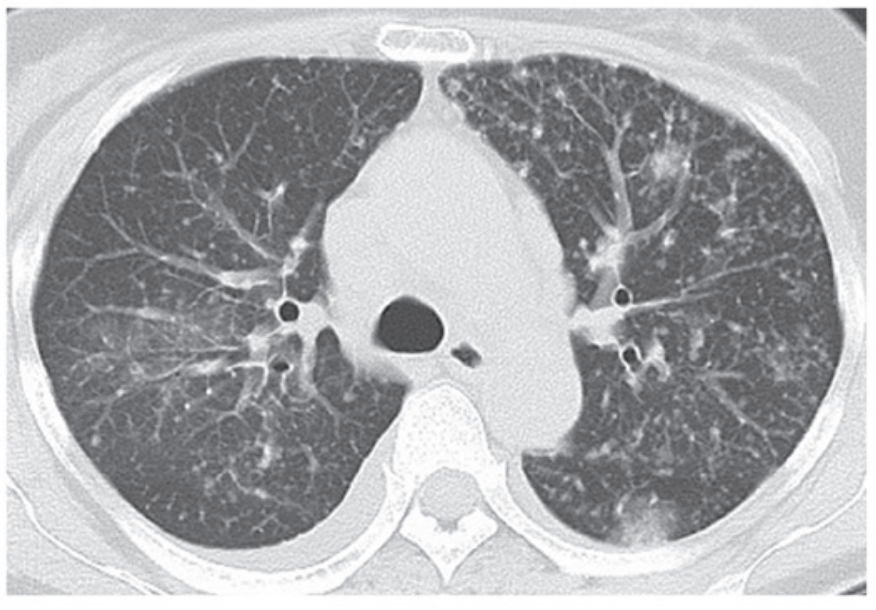

B

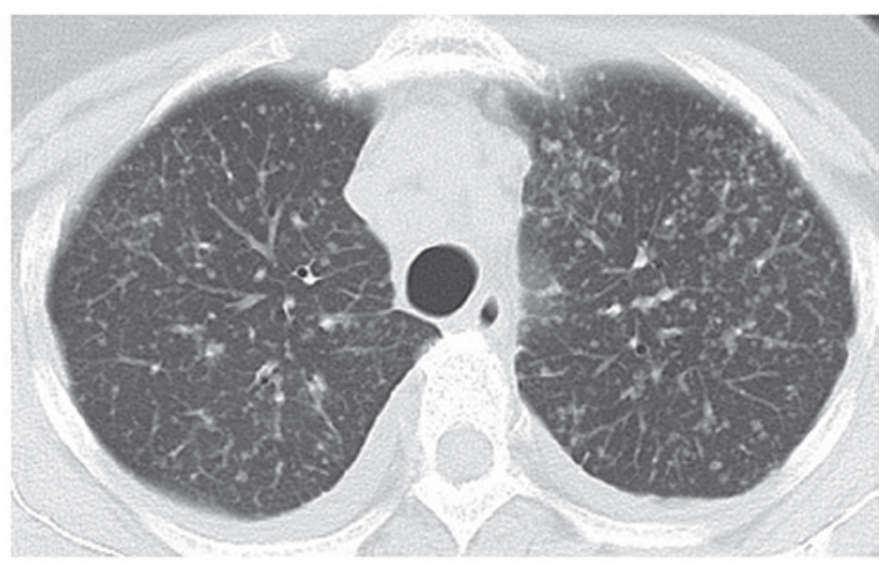

D

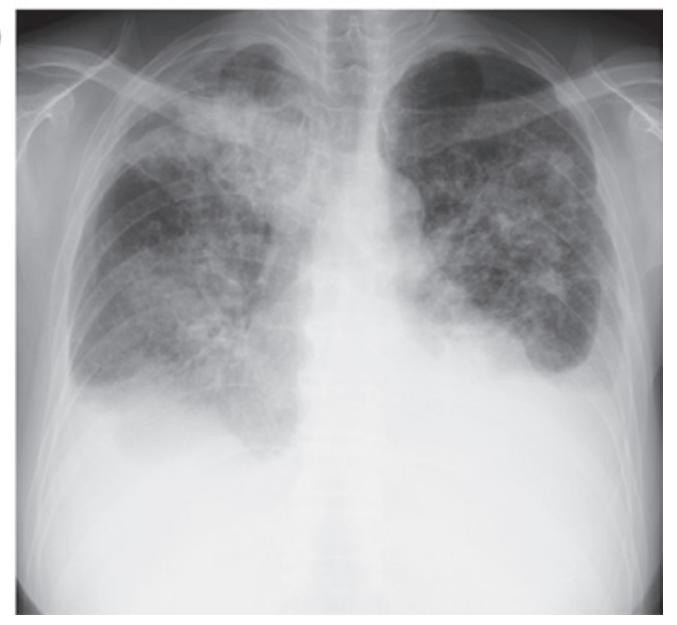

Figure 2. (A) Chest radiograph showed multiple small nodules bilaterally in the lungs. (B and C) Chest CT demonstrated numerous tiny nodules throughout bilaterally in the lungs with some consolidations. (D) The consolidation in the right upper-lung field developed with bilateral pleural effusion. 
Case 2. A 61-year-old non-smoking woman developed cough and dyspnea during exercise in December 2016. Consequently, she visited a hospital on January 142017. Because both chest radiograph and $\mathrm{CT}$ revealed miliary lung nodules (Fig. 2A-C) and miliary tuberculosis was suspected, she was transferred to our hospital on the same day. Because a sputum smear stain for mycobacteria was negative, transbronchial lung biopsy was performed on January 17 and micropapillary adenocarcinoma staged cT4N3M1b (PLE, PUL, OSS) was diagnosed. Furthermore, exon 20 insertion was detected by cobas version 2 method. Because the PS score was 1, carboplatin plus nab-paclitaxel were administered as first-line treatment on February 1. However, this therapeutic regimen did not produce a satisfactory outcome (Fig. 2D). Therefore, we administered docetaxel as second-line therapy on February 23 but the outcome was the same as that produced by first-line treatment, and the patient rapidly developed respiratory failure. Finally, she died of lung cancer on March 17.

\section{Discussion}

The results of our two cases would provide the following two important clinical issues.

First, NSCLC with exon 20 insertion can present miliary type of lung metastases. In fact, two in four NSCLC patients (with exon 20 insertion) (50\%) had miliary lung metastases. To the best of our knowledge, there is no report regarding the radiological characteristics of patients with NSCLC with exon 20 insertion. Furthermore, it is widely accepted that NSCLC with major EGFR mutations, especially exon 19 deletion, occasionally presents a miliary metastatic pattern in lungs. Laack et al reported five cases of miliary lung metastases from NSCLC with exon 19 deletion, which dramatically responded to EGFR-TKIs (10). In addition, two recent studies by Togashi et al and $\mathrm{Wu}$ et al revealed that NSCLC patients with miliary lung metastases had a high EGFR mutation rate in the range of $70-87.5 \%(3,4)$. However, in these studies, exon 20 insertions were not observed (4), and the details of EGFR mutation status were not provided (3). Most of EGFR mutations were major EGFR mutation (4). More recently, miliary type of metastases in lung and/or brain was reported in NSCLC harboring non-EGFR oncogene driver mutation such as echinoderm microtubule associated protein like 4-anaplastic lymphoma kinase (EML4-ALK) gene translocation (11) and c-ros oncogene 1 (ROS-1) rearrangement (12). Taking together these reports and the two cases presented in this study, miliary type of metastases can be closely correlated not only with major EGFR mutation, but also other oncogene driver mutation including exon 20 insertion.

Second, miliary lung metastases from NSCLC with exon 20 insertion appear to be an extremely poor prognostic entity. As reported in this study, disease progression in the two patients was rapid, and both the patients died within 3 months from beginning first-line treatment. To date, the prognosis in NSCLC patients with exon 20 insertions has been reported to vary, with a median OS of 5-26 months (7,8,13-15). However, most studies included a high proportion of patients with post-operation recurrence, which suggests low-tumor burden.
In contrast, a study by Noronha et al included more patients with $\mathrm{PS} \geq 3$ (40\%) and revealed a dismal poor prognosis, with a median OS of 5 months (8). The authors proposed that the reasons for poor prognosis were attributable to high tumor burden and poor response to EGFR-TKIs (8), which were observed in the prospective randomized trial (16). In the two cases presented in this study, despite high tumor burden, when the first-line treatment was commenced, the PS score was low ( 0 for the first and 1 for the second case). However, miliary lung metastases easily and rapidly caused respiratory failure resulting in PS reduction. Therefore, NSCLC patients with miliary lung metastases and exon 20 insertions seem to have a poor prognosis with low chances of receiving further chemotherapy.

In conclusion, we presented two cases of miliary lung metastases from NSCLC patients with exon 20 insertion. PS rapidly deteriorated and the efficacy of chemotherapies including cytotoxic agents and EGFR-TKIs was limited. Chest physician should be aware that NSCLC with exon 20 insertion could present as miliary type of lung metastases and rapid disease progression. The accumulation of additional case series is necessary to confirm our results.

\section{Acknowledgements}

Not applicable.

\section{Funding}

No funding was received.

\section{Availability of data and materials}

The datasets used and/or analyzed during the current study are available from the corresponding author on reasonable request.

\section{Authors' contributions}

AS and HS participated in the conception and design of the case report, analyzed and interpreted the data and wrote the manuscript. AS, TaK, TsO, SI and $\mathrm{TaO}$ evaluated the patient and participated in the therapy. SI, HS and $\mathrm{TaO}$ revised the manuscript for intellectual content. TI, ToK, and KO evaluated radiological images or pathological specimens. All authors have read and approved the final draft.

\section{Ethics approval and consent to participate}

The patient provided written informed consent for participation in the present study.

\section{Patient consent for publication}

The patient provided written informed consent for the publication of any associated data and images in this case report.

\section{Competing interests}

The authors declare that they have no competing interests. 


\section{References}

1. Lynch TJ, Bell DW, Sordella R, Gurubhagavatula S, Okimoto RA, Brannigan BW, Harris PL, Haserlat SM, Supko JG, Haluska FG, et al: Activating mutations in the epidermal growth factor receptor underlying responsiveness of non-small-cell lung cancer to gefitinib. N Engl J Med 350: 2129-2139, 2004.

2. Paez JG, Janne PA, Lee JC, Tracy S, Greulich H, Gabriel S, Herman P, Kaye FJ, Lindeman N, Boggon TJ, et al: EGFR mutations in lung cancer: Correlation with clinical response to gefitinib therapy. Science 304: 1497-1500, 2004.

3. Togashi Y, Masago K, Kubo T, Sakamori Y, Kim YH, Hatachi Y, Fukuhara A, Mio T, Togashi K and Mishima M: Association of diffuse, random pulmonary metastases, including miliary metastases, with epidermal growth factor receptor mutations in lung adenocarcinoma. Cancer 117: 819-825, 2011.

4. Wu SG, Hu FC, Chang YL, Lee YC, Yu CJ, Chang YC, Wu JY, Shih JY and Yang PC: Frequent EGFR mutations in NSCLC presenting with miliary intrapulmonary carcinomatosis. Eur Respir J 41: 417-424, 2012.

5. Sekine A, Kato T, Hagiwara E, Shinohara T, Komagata T, Iwasawa T, Satoh H, Tamura K, Kasamatsu T, Hayashihara K, et al: Metastatic brain tumors from non-small cell lung cancer with EGFR mutations: Distinguishing influence of exon 19 deletion on radiographic features. Lung Cancer 77: 64-69, 2012.

6. Mochizuki S, Nishimura N, Inoue A, Murakami K, Nukiwa T and Chohnabayashi N: Miliary brain metastases in 2 cases with advanced non-small cell lung cancer harboring EGFR mutation during gefitinib treatment. Respir Investig 50: 117-121, 2012.

7. Naidoo J, Sima CS, Rodriguez K, Busby N, Nafa K, Ladanyi M, Riely GJ, Kris MG, Arcila ME and Yu HA: Epidermal growth factor receptor exon 20 insertions in advanced lung adenocarcinomas: Clinical outcomes and response to erlotinib. Cancer 121: 3212-3220, 2015.

8. Noronha V, Choughule A, Patil VM, Joshi A, Kumar R, Susan Joy Philip D, Banavali S, Dutt A and Prabhash K: Epidermal growth factor receptor exon 20 mutation in lung cancer: Types, incidence, clinical features and impact on treatment. Onco Targets Ther 10: 2903-2908, 2017.
9. Yasuda H, Kobayashi S and Costa DB: EGFR exon 20 insertion mutations in non-small-cell lung cancer: Preclinical data and clinical implications. Lancet Oncol 13: e23-e31, 2012.

10. Laack E, Simon R, Regier M, Andritzky B, Tennstedt P, Habermann C, Verth CZ, Thöm I, Grob T, Sauter G and Bokemeyer C: Miliary never-smoking adenocarcinoma of the lung: Strong association with epidermal growth factor receptor exon 19 deletion. J Thorac Oncol 6: 199-202, 2011.

11. Falk AT, Poudenx M, Otto J, Ghalloussi H and Barrière J: Adenocarcinoma of the lung with miliary brain and pulmonary metastases with echinoderm microtubule-associated protein like 4-anaplastic lymphoma kinase translocation treated with crizotinib: A case report. Lung Cancer 78: 282-284, 2012.

12. Dziadziuszko K, Szurowska E, Pienkowska J, Jassem J and Dziadziuszko R: Miliary brain metastases in a patient with ROS1-rearranged lung adenocarcin-oma: A case report. J Thorac Oncol 9: e34-e36, 2014.

13. Oxnard GR, Lo PC, Nishino M, Dahlberg SE, Lindeman NI, Butaney M, Jackman DM, Johnson BE and Jänne PA: Natural history and molecular characteristics of lung cancers harboring EGFR exon 20 insertions. J Thorac Oncol 8: 179-184, 2013.

14. Lund-Iversen M, Kleinberg L, Fjellbirkeland L, Helland $\AA$ and Brustugun OT: Clinicopathological characteristics of 11 NSCLC patients with EGFR-exon 20 mutations. J Thorac Oncol 7: 1471-1473, 2012.

15. Beau-Faller M, Prim N, Ruppert AM, Nanni-Metéllus I, Lacave R, Lacroix L, Escande F, Lizard S, Pretet JL, Rouquette I, et al: Rare EGFR exon 18 and exon 20 mutations in non-small-cell lung cancer on 10117 patients: A multicentre observational study by the French ERMETIC-IFCT network. Ann Oncol 25: 126-131, 2014.

16. Yang JC, Sequist LV, Geater SL, Tsai CM, Mok TS, Schuler M, Yamamoto N, Yu CJ, Ou SH, Zhou C, et al: Clinical activity of afatinib in patients with advanced non-small-cell lung cancer harbouring uncommon EGFR mutations: A combined post-hoc analysis of LUX-Lung 2, LUX-Lung 3 and LUX-Lung 6. Lancet Oncol 16: 830-838, 2015. 